\title{
Filigrane
}

Écoutes psychothérapiques

\section{Une temporalité qui va d'un écrit de 1920 à sa publication en 2009}

\section{Janine Altounian}

Volume 22, numéro 2, automne 2013

Psychanalyse et temporalités II

URI : https://id.erudit.org/iderudit/1022553ar

DOI : https://doi.org/10.7202/1022553ar

Aller au sommaire du numéro

Éditeur(s)

Revue Santé mentale au Québec

ISSN

1192-1412 (imprimé)

1911-4656 (numérique)

Découvrir la revue

Citer cet article

Altounian, J. (2013). Une temporalité qui va d'un écrit de 1920 à sa publication en 2009. Filigrane, 22(2), 9-26. https://doi.org/10.7202/1022553ar
Résumé de l'article

L'article se propose d'illustrer, par un exemple personnel, la pertinence d'une gestation tributaire du temps - temps psychique, temps généalogique, temps historique des événements sociopolitiques - grâce auquel un écrit testamentaire de 1920 finit par trouver sa véritable destination dans une publication de 2009. Pour montrer comment exhumer une trace, faire entendre une voix, requiert plusieurs générations, il présente les différentes étapes que dut franchir le témoignage de déportation de Vahram Altounian, survivant du génocide arménien de 1915, jusqu'à sa parution en fac similé dans une édition universitaire au sein d'un ensemble d'élaborations dont il fut le référent pour sept récipiendaires : son traducteur, sa fille et cinq psychanalystes à l'écoute des traumas de l'Histoire.
Ce document est protégé par la loi sur le droit d'auteur. L'utilisation des services d’Érudit (y compris la reproduction) est assujettie à sa politique d'utilisation que vous pouvez consulter en ligne.

https://apropos.erudit.org/fr/usagers/politique-dutilisation/ 


\title{
Une temporalité qui va d'un écrit de 1920 à sa publication en 2009
}

\author{
Janine Altounian
}

L'article se propose d'illustrer, par un exemple personnel, la pertinence d'une gestation tributaire du temps - temps psychique, temps généalogique, temps historique des événements sociopolitiques - grâce auquel un écrit testamentaire de 1920 finit par trouver sa véritable destination dans une publication de 2009. Pour montrer comment exhumer une trace, faire entendre une voix, requiert plusieurs générations, il présente les différentes étapes que dut franchir le témoignage de déportation de Vahram Altounian, survivant du génocide arménien de 1915, jusqu'à sa parution en fac similé dans une édition universitaire au sein d'un ensemble d'élaborations dont il fut le référent pour sept récipiendaires: son traducteur, sa fille et cinq psychanalystes à l'écoute des traumas de I'Histoire.

\section{S} i certains écrivains témoins n’ont pu écrire que longtemps après les événements inouïs auxquels ils survécurent (p. ex. Jorge Semprun, Jean Améry, Charlotte Delbo, Imre Kertész, Ruth Klüger) en opérant un clivage dans l'espace de leur vie psychique, cette gestation intrapsychique du temps est comparable à celle, transgénérationnelle, d'une filiation qui se constitue, elle aussi, d'une coupure puisqu'elle opère, chez l'héritier de celui qui est devenu apatride, une sorte de marcottage dans une terre nouvelle.

Aux écrivains témoins était nécessaire la création de la distance et des instances médiatrices au sein de leur monde interne afin de pouvoir s'éloigner quelque peu de ce à quoi ils avaient survécu. Un long temps devait s'écouler pour que naissent en eux des forces de survie génératrices d'enveloppes psychiques susceptibles d'accueillir dans l'écriture des souvenirs terrifiants, souvenirs malgré tout non partageables avec ceux d'ici, restés indemnes d'un meurtre de l'humain, donc meurtre d'une partie d'eux-mêmes. Mais il en va de même dans le temps transgénérationnel qui s'écoule du survivant à ses descendants. Les deux configurations temporelles relèvent d'une même renaissance des pulsions de vie qui, étonnamment, réclament d'être réinvesties, soit pour témoigner des chers disparus, abandonnés sans traces, soit pour 
enfanter ceux à qui cette tâche sera inconsciemment déléguée (Waintrater, 2003, 186).

J'illustrerai par un exemple personnel la pertinence de cette gestation tributaire du temps - temps psychique, temps généalogique, temps historique des événements sociopolitiques, donc temps excédant les limites de la vie individuelle. Celui-ci présentera notamment les différentes étapes que dut franchir le témoignage de déportation de Vahram Altounian, survivant du génocide arménien, écrit en 1920, jusqu'à sa parution en 2009 en facsimile dans une édition universitaire au sein d'un ensemble d'élaborations dont il fut le référent pour sept récipiendaires: son traducteur, sa fille et cinq psychanalystes à l'écoute des traumas de l'Histoire (V. et J. Altounian, 2009). Le facteur temporel instaurant donc une pluralité de liens, non seulement verticaux mais également horizontaux, la constitution de ce regroupement confirme, d'une part, ce que soutient un des auteurs analysant ce récit:

Pour se penser, écrit-il, la catastrophe doit être échoïsée, amplifiée, susciter témoignages et commentaires, convoquer de multiples versions. (Kaës, 2009, 210)

D'autre part, l'incipit de cet ouvrage, «co-signé par une vivante et un mort.»(Trévisan, 2009, 16), attire ainsi l'attention du lecteur sur sa genèse transgénérationnelle:

Cet ouvrage, dont le contenu pourrait s'intituler: «Le génocide dans le Journal d'un père et dans la mémoire de sa fille» n'a pu se constituer que par un travail d'écriture qui a nécessité deux générations et plusieurs voix pour voir le jour et s'inscrire dans ces pages. Nous avons retranscrit cette écriture en deux temps en l'attribuant à Vahram et Janine Altounian. (V. et J. Altounian, 2009, 5)

Nous verrons dans quelles circonstances cette cosignature, pour le moins troublante, s'imposa à une telle réception polyphonique qui se propose de rendre compte, en et par ce rassemblement, de la transmission d'un héritage traumatique. 
Mais j'aimerais auparavant témoigner de cette "nouvelle temporalité [...] qui fait fi du temps chronologique» évoquée par l'argument de ce numéro de Filigrane, en montrant combien celle-ci peut inviter un analysant à « retrouver le temps d'autrefois resté intact », je dirais même, d'un autrefois que lui-même n'a pas vécu ${ }^{1}$. Une expérience relativement récente m'a fait notamment ressentir des effets de lecture rendus possibles grâce au «hasard » après un grand nombre d'années, lors de la levée d'un refoulement qui avait frappé, jusque là, des récits pourtant bien connus de moi. Lorsque mes doigts se mirent à suivre à la trace les énonciations du témoin-scripteur qu'avait été mon père, l'interposition de ma main écrivant sur un clavier d'ordinateur entre la lectrice copiste que j'étais et des récits familiaux ghettoïsant, créait une distance qui favorisait la perception d'horribles scènes tout en révélant ainsi le refoulement qui les avait mises à l'écart.

En effet, comme le futur ouvrage collectif (V. et J. Altounian, 2009) autour de la traduction du Journal de déportation de mon père exigeait d'en avoir une version électronique ${ }^{2}, \mathrm{j}^{\prime}$ avais fait sciemment le choix de la recopier moi-même alors que la solution de facilité aurait été de la scanner. Je tenais à reparcourir le douloureux cheminement du père-adolescent en l'accompagnant patiemment de ma main, pas à pas, mot après mot. Me retrouvant ainsi, pendant la frappe, devant la réalité que déchiffrait chaque phrase de cette narration, je me suis aperçu que, malgré mes innombrables lectures, je ne l'avais lue qu'en l'évitant, n'en avais pas épuisé les affects empêchés qu'elle recelait et dont elle me chargeait encore maintenant de tant de larmes non versées. Le refoulement avait donc bien ouvré, dicté par le désir de ne plus entrouvrir la porte dévoilant ces espaces effrayants.

Pendant ce travail de lectrice copiste, je vécus tous mes jours en 1915 et 1916, en compagnie de ce garçon d'à peine quatorze ans, seul soutien après la mort du père d'une mère résistante et digne. Ce couple uni face à l'impératif de rester en vie me transportait dans des déserts où soufflaient la terreur, le meurtre, l'imminence du mourir. Je me retrouvais entièrement dans ces années, ces contrées-là, j'avais peur de voir ce qui allait survenir, peur de ce que les mots allaient écrire sur l'écran de l'ordinateur et, au bout de ma copie où « en novembre 1919 on a pris le bateau et on est arrivé à Marseille sept jours plus tard, c'est-à-dire en décembre », je débarquais avec ces immigrants, délivrée enfin de ma peur, en terre de France. C'est alors que, rassurée par notre retour, délivrée de cette menace des mots surgissant sur l'écran, j'eus le courage d'examiner attentivement les papiers échoués de lieux et de temps si lointains dans l'enveloppe contenant le mystérieux cahier du manuscrit. Je 
découvris deux lettres que je m'étais évertuée à ignorer jusqu'alors. Je les fis traduire (V. et J. Altounian, 2009, 42-46). Elles étaient écrites, juste trois et quatre mois avant notre arrivée à Marseille, par mes oncles vivant déjà en France; la première, de juin 1919, était une sorte d'avis de recherche expédié de Lyon et la seconde du mois suivant s'adressait à la «Très vénérable grand-mère, chère mère Nahidé», retrouvée enfin vivante. En les lisant, je compris que j'avais eu encore besoin de la voix de ces contemporains pour pouvoir insérer dans une temporalité objective le texte que je venais de taper. "Douce mère, disait la dernière lettre, bien que nous n'ayons pas de nouvelles de vous depuis quatre ans... " Ce fut seulement alors que je réalisai la durée de quatre ans des épreuves de là-bas, alors que s'éprouvait après coup en moi, l'angoisse vécue ici, à Lyon, de ces quatre années sans nouvelles, angoisse qui allait bientôt se transformer en certitude qu'aucun avis de recherche ne ferait revenir le père. C'était donc encore une fois le refoulement qui m'avait éloignée d'aller voir ce que contenaient ces lettres menaçantes.

Pour essayer de comprendre ce refoulement obstiné — symptôme d'incrédulité inconsciente dans mon cheminement progressif vers le point focal des charniers du désert —, j'aimerais m'arrêter à quelques lignes de Freud (1936, 331-338) qui, dans un contexte différent mais référé, en dernier ressort, à une relation au père, démasque l'incrédulité comme source d'un «trouble » de la connaissance:

Lorsque $[\ldots]$ je me trouvai sur l'Acropole et que mon regard embrassa le paysage, il me vint subitement cette curieuse pensée : ainsi donc tout cela existe effectivement comme nous l'avons appris à l'école?! [...] comme quelqu'un qui, se promenant en Écosse sur les bords du Loch Ness, verrait tout à coup devant soi, apporté sur le rivage par le flot, le corps du monstre dont on a tant parlé et qui se trouverait contraint à avouer: Il existe donc effectivement, le serpent de mer auquel nous n'avons pas cru! [...] Une telle incrédulité est manifestement une tentative pour récuser un morceau de la réalité [...] Je ne me souviens pas simplement d'avoir dans mes jeunes années douté de jamais voir un jour l'Acropole moi-même, mais j'affirme qu'alors je n'ai absolument pas cru à la réalité de l'Acropole [...] Sur l'Acropole j'avais eu — ou aurais pu avoir — pour un moment ce sentiment: ce que je vois là n'est pas effectif.

Pour dénoncer en le parodiant un mouvement psychique analogue qui dut s'esquisser en moi, je dirais qu'en lisant le témoignage de mon père 
quelque chose en moi devait s'être dit: «ce que je lis là n'est pas effectif», ou de façon plus insidieuse : "ce que je lis là ne me concerne pas effectivement », mais c'est en écrivant de ma main les mots de son texte que j'avais réellement "vu tout à coup devant moi, apporté sur le rivage par le flot [de l'Euphrate], le corps du monstre dont on [m'avait] tant parlé et [...] auquel [je n'avais] pas cru».

Freud en arrive alors à la conclusion:

[Lors de ces phénomènes] nous apparaît comme étranger ou bien un morceau de la réalité, ou bien un morceau du moi propre. Dans ce dernier cas, on parle de «dépersonnalisation » [...] ils servent tous à la défense, voulant tenir à distance du moi quelque chose, le dénier.

J'en conclus donc que, lors de mes très nombreuses lectures du manuscrit paternel, j'avais encore voulu « tenir à distance du moi quelque chose, le dénier» en tant qu'impliquant ma propre existence actuelle. Or, je ne suis pas sans remarquer que, pour illustrer ces phénomènes de dépersonnalisation, Freud prend un exemple mettant en jeu sa relation au père:

Notre père avait été commerçant, il n'avait pas reçu la formation du lycée, Athènes ne pouvait pas signifier grand-chose pour lui. Ce qui nous troublait dans la jouissance du voyage à Athènes était donc une motion de piété.

Mon père, lui, avait été artisan ainsi que beaucoup de survivants arméniens. Comme celui de Freud, «il n'avait pas reçu la formation du lycée», mais si je me permets de rapprocher ces deux situations différentes de «dépersonnalisation », c'est en raison de leur cause commune : la «motion de piété», «Regung der Pietät» envers le père, comme l'écrit Freud à Romain Rolland en recourant à ce terme de racine latine, «Pietät», extrêmement rare dans le corpus de son œuvre. Or si le «trouble du souvenir» de Freud était dû à une «motion de piété», le déni de la réalité, dans mon «trouble» de lecture, était certes dû au «moi» menacé, refoulant de la lectrice, mais plus précisément sans doute à la "piété » vis à vis de sa représentation d'un père.

Je retracerai donc un itinéraire emblématique où, à partir d'un écrit laissé ignoré au fin fond d'une armoire, le témoignage d'une expérience 
traumatique débutant à Boursa, petite ville d'Asie mineure, "un mercredi 10 août 1915 », est passé par l'épreuve de sa traduction, de son élaboration subjective par un héritier, pour se transmettre et aboutir, quasi un siècle plus tard, à une lisibilité partagée au sein de cette ultime réception collective.

Pour introduire mon propos, je citerai un philosophe, Walter Benjamin qui, se référant à la transmission d'une catastrophe historique, nomme « expérience » ce qui ne peut se transmettre à l'héritier d'un meurtre de masse en fait de richesse et de sens pour sa propre vie. À propos des « revenants» de la Grande Guerre qui furent réduits au silence, il rappelle que :

Le cours de l'expérience a chuté, et ce dans une génération qui fit en 19141918 l'une des expériences les plus effroyables de l'histoire universelle [...] N'a-t-on pas alors constaté que les gens revenaient muets du champ de bataille? Non pas plus riches, mais plus pauvres en expérience communicable. (Benjamin, 2000, 365)

Ces mots du philosophe s'appliquent effectivement au manque de parole entre mon père et moi sur ce que put être son expérience d'un génocide perpétré justement sur le versant oriental de la Guerre de $14^{3}$ et dont le compte rendu qu'il en fit s'intitule précisément: «10 août 1915, mercredi : tout ce que j'ai enduré, des années 1915 à 1919 ». C'est en fait le destin posthume de ce Journal que va évoquer le présent exposé dont le titre pourrait s'énoncer: «Exhumer une trace, l'inscrire, témoigne d'un acte de résistance qui requiert plusieurs générations». Le sens que ce manuscrit «restaure», celui notamment d'un attachement irréductible du sujet à son héritage et à son identité, renvoie certes à la scène princeps de l'inhumation du père, mais surtout aux avatars que connut, à la génération suivante, cette mise en terre du père qui appela plus tard son expression symbolique dans la mise en mots du fils. Cette restauration sera en somme celle des liens affectifs, culturels, politiques qui, dans une transmission unissent les générations entre elles.

Dans l'essai précédemment cité de Benjamin, «Expérience et pauvreté», le philosophe dénonçait la rupture, consécutive à la Grande Guerre, de cette transmission de l'expérience. Or, pour montrer ce que serait, au contraire, son maintien d'une génération à l'autre, il commence par raconter cette fable que notre cher La Fontaine nous avait déjà apprise sur les bancs de l'école:

Dans nos manuels de lecture figurait la fable du vieil homme qui sur son lit de mort fait croire à ses enfants qu'un trésor est caché dans sa vigne. Ils 
n'ont qu'à chercher. Les enfants creusent, mais nulle trace de trésor. Quand vient l'automne, cependant, la vigne donne comme aucune autre dans tout le pays. Ils comprennent alors que leur père a voulu leur léguer le fruit de son expérience : la vraie richesse n'est pas dans l'or, mais dans le travail. [...] Où les mourants prononcent-ils encore des paroles impérissables, qui se transmettent de génération en génération comme un anneau ancestral? (Benjamin, 2000, 365)

S'il faut mettre en lien le motif du travail avec celui de la transmission que Benjamin spécifie ici prioritairement par une transmission qui prône la valeur du travail humain, c'est bien sûr parce que, au delà de l'extermination des hommes, les régimes totalitaires visent l'anéantissement de ce qu'a produit la créativité de ce travail, et la ruine des attachements des hommes entre eux et à leur histoire. Les nazis n'inscrivirent-ils pas aux portes de leurs camps l'insulte de cette antiphrase profanatrice et criminelle: «Arbeit macht frei» («Le travail rend libre») ? Il convient également de rapprocher ces deux motifs de la transmission et du travail parce qu'hériter pour vouloir témoigner réclame la prise en charge d'un travail. Hériter se fait par un travail. Ces vers de Gothe, cités en adage par Freud $(1912,379)$ comme modalité de transmission psychique le disent admirablement:

Ce que tu as hérité de tes pères, acquiers-le afin de le posséder, ce qu'on n'utilise pas est un pesant fardeau ${ }^{4}$.

La résistance d'un héritier de survivant est donc un travail d'appropriation de son héritage, le rendant capable de l'inscrire dans le monde des vivants. Voici quelques extraits du témoignage dont nous allons raconter le destin. Leur rythme, leur style sont très hachés car j’y ai procédé à de nombreuses coupures:

À Haman $[\ldots]$ nous avons constaté que les gens mangeaient des sauterelles. Des mourants, des morts partout $[\ldots]$ Mon père était très malade $[\ldots]$ bientôt il n'y a plus eu de sauterelles, car tout le monde en avait mangé. Et la déportation n'en finissait pas [...] Ma mère a dit: «Notre malade est très gravement atteint et partira la prochaine fois» $[. .$.$] «Vous osez parler?» a$ dit un gendarme et il a frappé à la tête de mon père. Ma mère suppliait $[\ldots]$ qu'on la frappe, elle, et qu’on laisse mon père. Sur ce, le gendarme a frappé 
ma mère [...] Six jours plus tard, le jour de la mort de mon père, ils ont de nouveau déporté. Ils frappaient notre mère. Nous deux frères, nous pleurions. Nous ne pouvions rien faire, car ils étaient comme une meute de chiens. Ils disaient à ma mère : «Ton malade est mort ». Et ma mère : « Nous partirons quand nous aurons enterré le mort». Ils répliquaient : «Non vous ferez comme les autres ». Les autres [...] abandonnaient les morts et la nuit les chacals les dévoraient. J'ai vu que ça n'allait pas et qu'il fallait faire quelque chose. J'ai pris un flacon de 75 dirhem [ 1 dirhem $=3$ gr.], je l'ai rempli d'huile de rose et je suis allé voir le chef des gendarmes de la déportation [...] Nous sommes restés encore un jour. Nous avons creusé une fosse et nous avons payé cinq piastres au curé. Ainsi nous avons enterré mon père [...] Quinze jours après la déportation a recommencé [...] Ils brûlaient tout [...] Je me suis caché là, car j'ai su que plus loin ils tuaient les gens [...], on avait très faim et soif. J'ai vu que nous allions mourir de faim. [...] À Racca, on nous a montré une auberge. [...] Qu'avons nous vu? Les gens mouraient partout de faim. On ne pouvait pas rester à l'intérieur [...], tout sentait la pourriture [...] On n'avait pas d'argent, c'est pourquoi on a commencé à manger des herbes. [...] On a vu qu'on allait mourir. On faisait à peine deux pas et on tombait par terre. Ma mère a réfléchi: «Moi pour mourir, je mourrai, vous, il ne le faut pas!» C'est ainsi qu'elle nous a donnés, nous deux, aux Arabes. (V. et J. Altounian, 2009, 21, 24-25, 28)

C'est peu à peu que ce Journal paternel dont l'écriture me resta apparemment inconnue du vivant de son auteur acquit étrangement une vocation d'outre-tombe à témoigner. L'acte fondateur de scripteur qui avait animé ce père déterminait certes, à son insu, la transmission de sa mémoire et la reconstruction psychique du monde de sa fille, mais je doute que ce geste producteur de récits ultérieurs n'ait jamais été chez lui conscient de ses conséquences. Il est probable que ce Journal représenta simplement pour son rédacteur un moyen de poursuivre sa vie, une fois scellée dans un "petit cahier d'écolier» (V. et J. Altounian, 2009, 99), hors de lui, la recension des épreuves mortelles dont il ne fallait plus qu'il se souvienne. Si ce manuscrit contribua pour moi à une subjectivation aussi bien de mon histoire que de celle, après coup, du narrateur, son destin se joua en fait au moment où un événement politique parisien de septembre 1981, la prise d'otages au consulat de Turquie, vint réveiller en moi la réminiscence d'un souvenir d'enfance. 
Avant de décrire les différents moments d'épiphanie de ce manuscrit, je m’arrêterai d'abord sur cette réminiscence qui décida de sa première publication.

Ce fut la rencontre d'un fait objectif d'actualité avec la trace psychique d'une parole autrefois entendue, qui vint confirmer la temporalité transgénérationnelle des effets ce celle-ci, c'est-à-dire l'influence capitale qu'exerce sur le processus de transmission d'un héritage, aussi bien l'actualité d'événements contemporains, qu'une conception féconde de l'inconscient selon laquelle:

Rien ne peut être aboli qui n’apparaisse, quelques générations après [...] comme signe même de ce qui n'a pu être transmis dans l'ordre symbolique [...] La lettre parvient toujours à son destinataire même s'il n'a pas été constitué comme tel par le destinateur : la trace suit son chemin à travers les autres jusqu'à ce qu'un destinataire se reconnaisse comme tel. (Kaës, 1993,45)

En effet, lorsque cet acte dit «terroriste» éclata dans l'espace politique parisien, amorçant, en tant qu'acte de résistance, ce qu'on a appelé le « terrorisme publicitaire», lorsqu'il rompit dans ce qui était devenu «mon» pays un silence de plus d'un demi siècle sur le génocide arménien (J. Altounian, $2000,2-3)^{5}$ et interrogea alors un silence lové en moi, je sentis subitement que mon père aurait approuvé cet acte s'il avait été accompli de son vivant. Je retrouvai notamment, dans ma mémoire diffuse, le souvenir très lointain du plaisir de résistant avec lequel cet homme racontait à ses amis la prise, en 1896, de la Banque ottomane à Constantinople ${ }^{6}$. M'identifiant spontanément à la satisfaction avec laquelle il évoquait un exploit dont il était fier, je me sentis alors le droit de sortir de sa clandestinité protectrice cette relique que j'avais découverte.

Une semblable superposition temporelle d'un événement de 1981 réveillant le souvenir d'un récit paternel qui commémorait un événement de 1896 confirme le «rendez-vous tacite» dont Benjamin pose l'existence entre «les générations passées et la nôtre». Dans ses réflexions «Sur le concept d'histoire ", il développe notamment l'idée, parente de la conception freudienne de l'après-coup, selon laquelle le temps de la vie et le développement de l'être humain ne font que déployer, mettre au jour des points nodaux du passé qui contenaient déjà en eux tous ses éléments existant en germes.

Les voix auxquelles nous prêtons l'oreille n'apportent-elles pas un écho de voix désormais éteintes? [...] S'il en est ainsi, alors il existe un rendez-vous 
tacite entre les générations passées et la nôtre [...] À nous, comme à chaque génération précédente, fut accordée une faible force messianique sur laquelle le passé fait valoir une prétention. (Benjamin, 2000, 428)

Voici donc à présent la chronologie des étapes de cette transmission étrangement tenace, au cours de laquelle je pus hériter d'un récit, à vrai dire sans assignataire, une sorte de «bouteille à la mer » jetée par un père, un legs perçu, recueilli, porté à la traduction et publié par sa fille, une soixantaine d'années plus tard, après qu'un souvenir d'enfance eût pris ainsi tout son relief lors d'un événement politique.

En 1978, huit ans après le décès de mon père et au cours d'un travail analytique, je voulus explorer le milieu intellectuel arménien, lorsqu' «il me vint à l'idée ${ }^{7} »$ - peut-être à cause de l'angoisse que diffusait un savoir inconscient à ce sujet — que ma mère avait un jour mentionné avec un soupçon de désapprobation un manuscrit laissé par mon père. Sa dépréciation muette semblait dire: "Ah quoi bon avoir écrit puisque «ça » a eu lieu, que le monde l'a laissé faire et que nous avons tout perdu, à quoi bon retourner à ça!».

Je voulus le voir, elle alla le chercher.

Mais alors: que pouvait-on bien faire de cet objet redoutable, sacré? Avaisje le droit de toucher à ces pages si fragiles? Pour qui avaient-elles été écrites? À quelle fin? Dans quelles circonstances, quelle disposition d'esprit? Par quel hasard, quelle attention avaient-elles été conservées depuis tant d'années au fond d'une armoire? À qui poser ces questions? L'absence de toute médiation pour accompagner, introduire au monde des vivants ces feuilles angoissantes ne reproduisait-elle pas l'absence de tout protecteur auprès des orphelins qui, dans les déserts, avaient survécu à l'extermination de leurs parents? Ce cahier, un orphelin lui aussi, m'interrogeait, me demandait de me charger de lui. J'en avais peur comme d'une météorite tombée d'une autre planète mais j'en avais aussi pitié, je ne devais pas le laisser ainsi, inerte, seul dans un tel vide acoustique, muet. L'auteur de ce texte et de mes jours avait tenu à solliciter sa mémoire douloureuse pour raconter l'histoire d'une résistance à laquelle je devais ma naissance, que j'avais à honorer par l'acquittement d'une dette.

Je me mis en quête d'un traducteur: ce texte, pour moi hermétique, était écrit en caractères arméniens mais en langue turque (Beledian, 2009). Lorsque j'en reçus la version traduite, découvris brutalement ce que révélaient ces pages énigmatiques, déchiffrai en français ce qu'avait écrit cet homme que j’avais 
peu connu mais que je reconnaissais en tous points dans ses lignes, je basculai dans un état de suspension sans repères, une sorte de déréalisation qui me précipita dans une seconde analyse. Si ce passé terrifiant du parent survivant, pressenti à la maison, vécu en une sorte d'irréalité prudemment séparée de soi par clivage se présente à distance de lecture, écrit noir sur blanc dans la langue qui vous a appris la poésie et la pensée, sa réalité vous saute à la figure et c'est alors un effondrement où s'impose violemment à votre conscience ce qui relie votre temps présent à celui qui a connu ce versant hors humanité du monde, très peu d'années avant votre naissance, infiniment près de vous.

Disposant de cette traduction et retrouvant en moi, lors de l'événement scandaleux de 1981, le souvenir d'un père admiratif des résistants de 1896, je portai ce Journal aux Temps Modernes. Ils le publièrent (J. Altounian, V. Altounian, et Beledian, 1982) ${ }^{8}$, accompagné d'une postface et de notes indispensables de son traducteur, Krikor Beledian, mais également, de ma part, cette introduction où je relevais déjà ce qui, chez mon père adolescent, avait pu induire la décision ultérieure de rédiger son témoignage, peu après son arrivée en France en 1919 (Beledian, 2009):

Je retrouve dans ces pages une partie des récits qui ont peuplé mon enfance et celle de tous les Arméniens de mon âge. J’aimais passionnément écouter mon père, ses évocations brusquement évasives jetaient un voile sur les images insoutenables, mais la fermeture de son regard, la rétention de ces gestes, l'émotion secrète et la détermination de sa voix me parlaient d'un ailleurs qu'il avait, avant d'y être terrorisé, innocemment aimé. J’y entendais la nostalgie d'un pays dont il avait été, à jamais, arraché, abandonnant là, avec «nos maisons » et le corps du "père » enterré de justesse, les rêves de sa jeunesse, les racines de sa vie. Lorsque la distance entre la reconquête de chaque lecture refoule suffisamment l'afflux de l'émotion, pour donner place en moi à la lectrice profane et curieuse, je comprends le vif plaisir que je prenais enfant, à écouter l'auteur de ce journal, revivre étape après étape cette épopée d'où je suis, malgré tout, née. Il y avait quelque chose d'exaltant à ce que la vie - épouvante, humble nourriture encore une fois partagée ou marché aux couleurs d'espoir - soit une implacable aventure à déchiffrer et que survivre à l'oppression fut pour nous tous, Arméniens, un impératif incontournable. Pour la mémoire de ce grand-père enseveli je ne sais où, celle de tous les Arméniens dont ce compte rendu suggère, avec une sobriété troublante, le calvaire et la fin, pour honorer l'esprit de lutte et de résistance que l'adolescent de Boursa a dû puiser en lui afin de maintenir, dans les pires 
moments, la vie et son sens, j'ai cru être de mon devoir de rendre public son journal intime. Mon père, qui par ailleurs ne témoigna jamais de sympathie particulière pour la littérature, lui reprochant son impuissance, voire son ambiguité devant les impostures des puissants, a sans doute voulu, ici, par l'acte d'écrire, juguler, tenir à distance, exorciser la terreur « endurée». En fixant sur le papier l'incandescence de la mémoire, il essaya de temporiser le temps d'une génération. (J. Altounian, 1990, 83)

Ma famille et moi, nous avions ainsi bénéficié de plusieurs privilèges qui m'avaient permis d'exhumer une trace et de la faire parler:

- mon grand père assassiné avait pu, grâce à la "chance ", à la témérité de sa femme bravant les tueurs pour respecter un rituel de l'humanité et à la perspicacité de son fils adolescent, recevoir une sépulture et une prière;

- ce fils avait eu le courage de consigner scrupuleusement l'histoire de leur déportation;

— j'avais bénéficié d'une actualité qui m'autorisait à la publier ;

- et de l'instruction qui me permettait de le faire.

À l'occasion de la "publication» — dans tous les sens du mot — de ce manuscrit, qui dotait le tiers démocratique du pouvoir de donner toute sa portée à l'espace ouvert par l'acte politique, je pus revivre l'expérience salvatrice dont j'avais autrefois bénéficié à «L'École de la République» (J. Altounian, $2001 ; 2005,128$ sq.). Avec ses idéaux universalistes, celle-ci ignorait bien sûr l'histoire de la petite écolière arménienne que j'étais, mais - à condition qu'elle se soumette à ses apprentissages - elle lui accordait « démocratiquement » une place, une place à celle qui n'en avait aucune à la maison. Ainsi, il me fut rapporté que le manuscrit avait été qualifié de «texte sauvage» par Simone de Beauvoir et fait l'objet d'un certain doute de sa part. Néanmoins, elle le publia. Elle incarnait dans ma vie d'écolière, puis de femme, ces institutrices de l'École de Jules Ferry, (J. Altounian, 1990, 147), plutôt tolérantes à l'égard de ce qui les dépassait. «Faute de parler ma langue, l'arménien qui me parle, que je ne parle pas» (J. Altounian, 1990, 147) j'avais écrit:

Arménienne de France, j'avais dû en 1938, comme tant d'enfants de déracinés de nos jours, avec mes quatre ans, mes nattes rousses tressées par maman et mes yeux noirs aux aguets, émissaires du père, franchir le seuil de l'école maternelle, 7 rue de la Jussienne, comme on peut bien à cet âge affronter un pays étranger, menaçant, dont on comprend seulement qu'il est 
l'unique territoire des jours à venir. Les sombres forêts où sont abandonnées les petites filles des contes débouchent parfois sur de jolies clairières: j’y trouvai ces fées bienveillantes quoique trop assurées, mes institutrices, et devins peu à peu celle qui ne put traduire aux siens, en arménien, aucun de ces affects déchirants ou radieux qui initient au monde, aucun travail de la pensée, aucune évasion de l'imaginaire.

Une fois repris en 1990 dans mon premier livre, ce récit donna lieu à un incident totalement inattendu qui, en fait, eu pour conséquence cette troisième et ultime publication où, 90 ans après sa rédaction, il termina enfin son parcours insistant. À l'occasion d'une semaine de travail en 2007 avec des psychanalystes de Rome, l'une d'entre elles eu le désir de mettre en œuvre la publication d'un petit recueil italien (J. Altounian, V. Altounian, et Fraire, 2007) où figurait, entre autres, l'intégralité du récit paternel et un de mes articles qui le lui avait fait connaître. Lorsque j'eus le livre en main, je découvris d'abord avec stupeur et contrariété la désignation de l'auteur que l'éditeur n'avait pas jugé utile de préciser dans son contrat: celui-ci se nommait «Janine e Vahram Altounian». Après quelques instants, je finis non seulement par reconnaître que cette dénomination se justifiait totalement par le sous-titre du petit recueil, «Le génocide arménien dans le journal d'un père et la mémoire d'une fille», mais qu'une sorte d'événement psychique me parvenait là, de l'autre et de l'étranger, un événement qu'il fallait signifier en France par une publication du «même auteur bicéphale».

Cet écrit qui poursuivit en effet, au-delà de la mort de son scripteur, son désir de témoigner en provoquant un ultérieur «travail» d'écriture, de traduction, de publication fait penser à la notion de «livre 1 », telle que Luba Jurgenson (2003) définit un «texte écrit généralement tout de suite après le camp, dans l'urgence », en tant que "premier livre comme une mémoire prothèse » selon l'expression de Primo Levi. Lorsqu'elle déclare:

Il faut qu'une conversion s'opère pour que le survivant puisse prendre la parole, $[\ldots]$ l'oubli de l'expérience est la condition même du surgissement d'un texte,

on peut considérer que c'est dans l'ouvrage collectif, «livre 2 », que Vahram Altounian, 90 ans après son "écrit 1 », a pu "prendre la parole » au sein d'un regroupement d'auditeurs, l'expression «au sein» devant être prise ici à la lettre puisque non seulement le portage de ces six chercheurs et amis 
accompagne, soutient son témoignage devant le monde mais sa traduction, version dans laquelle ce récit avait jusque là été publié, se double ici du facsimile de sa version originale.

Il me faut ici préciser que ce fut l'éditeur lui-même, en l'occurrence le directeur des PUF, qui souhaita la reproduction vivante de ce témoignage à laquelle je n'aurais, moi, évidemment pas osé penser, que j'aurais encore moins osé réclamer dans une édition " universitaire» où le scripteur de ces terribles pages voisine avec les textes freudiens dont je suis cotraductrice depuis 1970 (J. Altounian, 2003) ${ }^{9}$. Cette insertion du facsimile du manuscrit que je dois, encore une fois, à une initiative démocratique républicaine, figure métaphoriquement l'inclusion, dans le monde de l'Histoire, du corps de ceux qui furent exclus de ce monde.

Cette inclusion est aussi le fruit de l'injonction à «travailler ", proférée par l'ancêtre et transmise jusqu'à sa petite fille qui lui rend ainsi hommage. Par sa présence sur fond de couleur sépia avec des pages aux bords abîmés par les ans, elle rend manifeste l'aboutissement tangible d'un «travail» d'exhumation qui aura duré trois générations.

Janine Altounian janine.altounian@free.fr

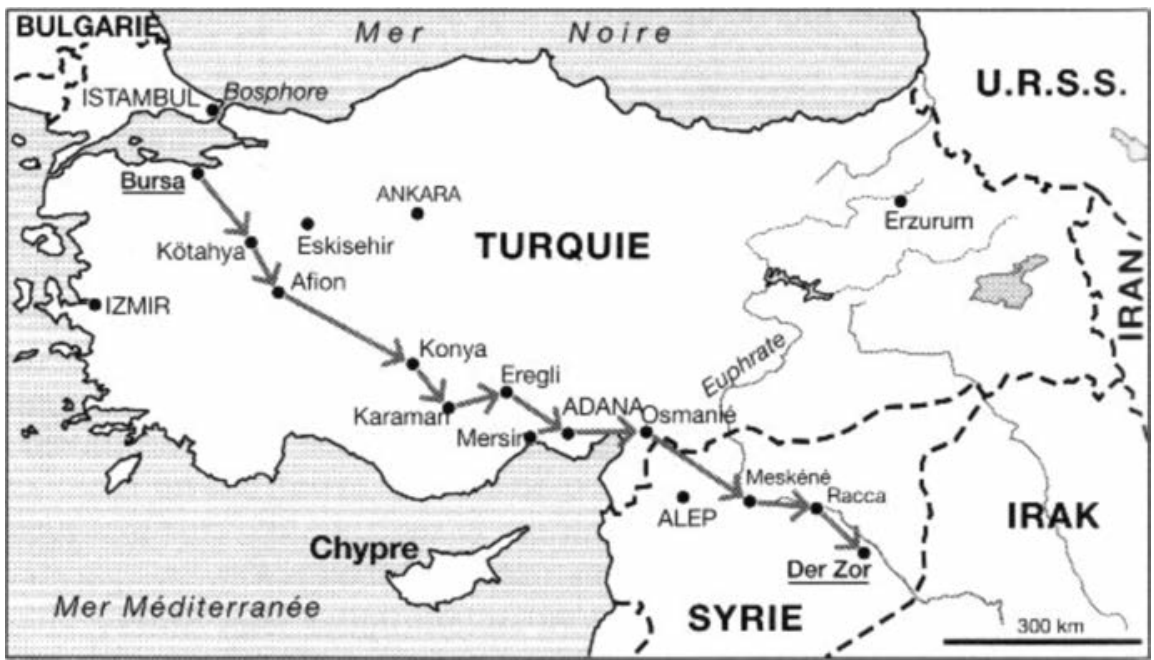

Itinéraire de déportation de Vahram Altounian, établi par son traducteur Krikor Beledian. 
Une temporalité qui va d'un écrit de 1920 à sa publication en 200923

\section{Lipar 10.}

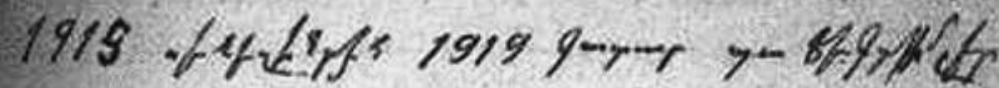

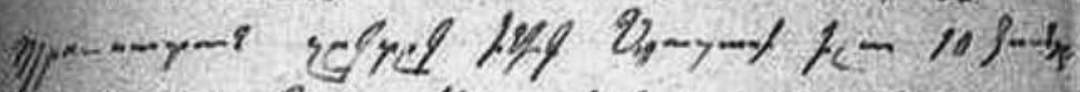
H.

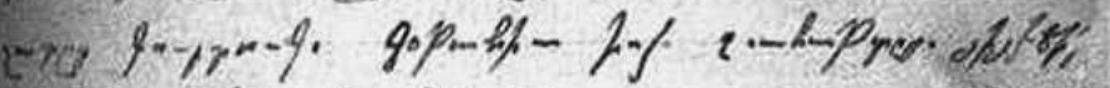

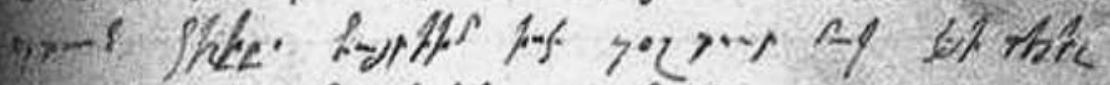

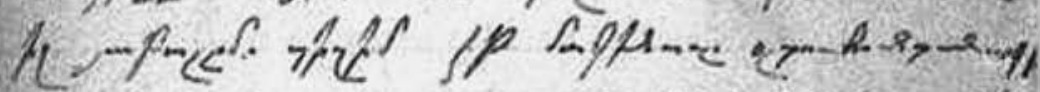

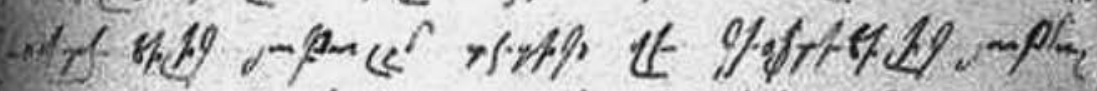

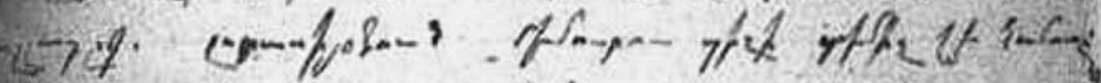

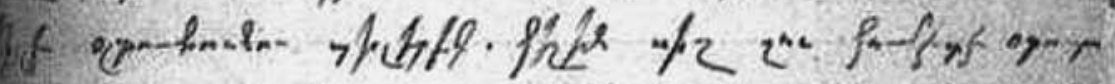

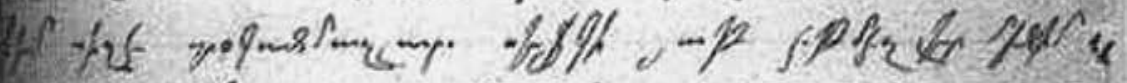

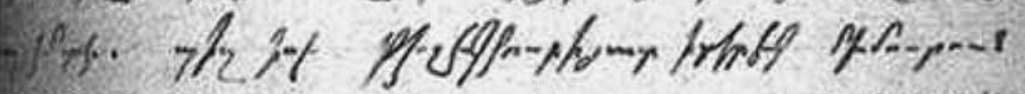

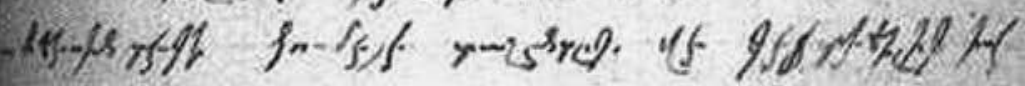

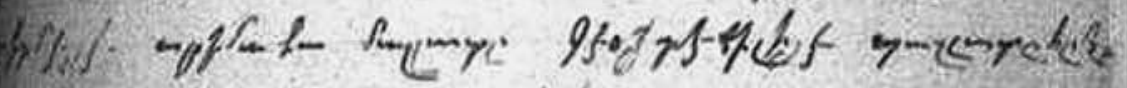

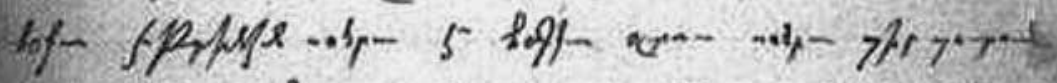

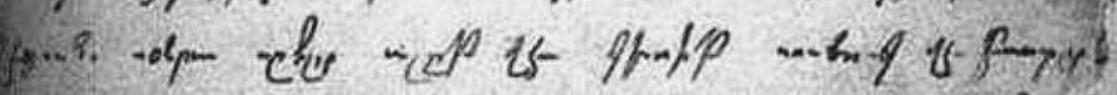

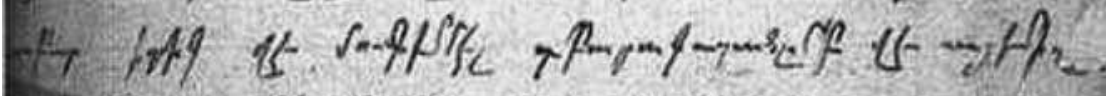

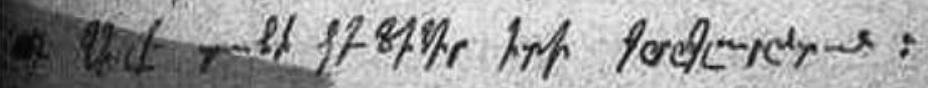

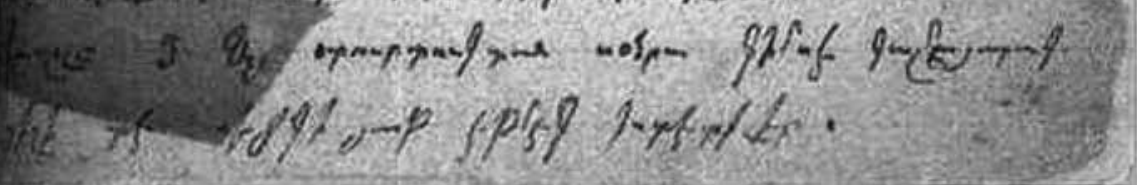




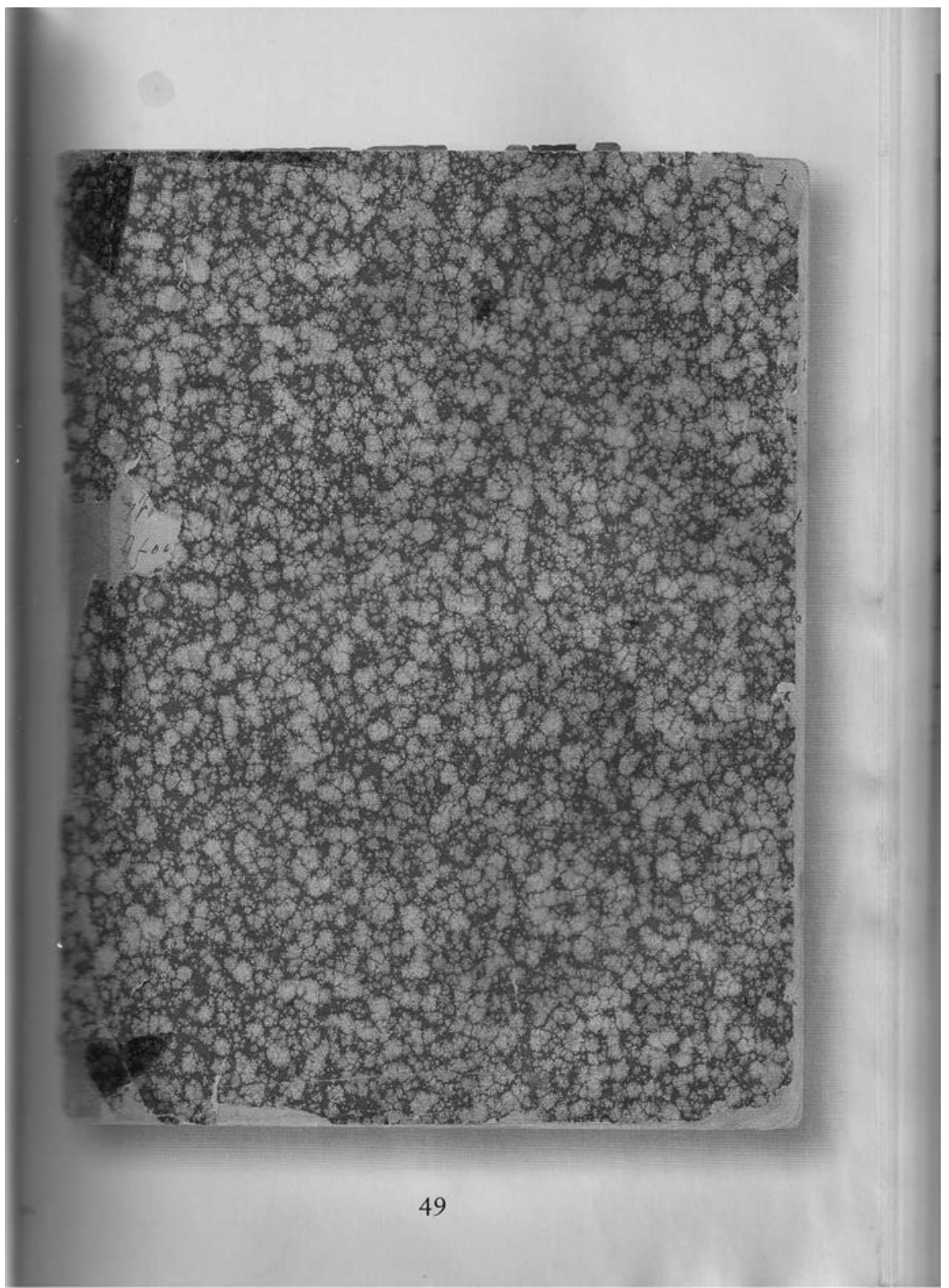

Couverture du petit cahier, tel qu'il est reproduit à la page 49 de Mémoires $d u$ Génocide arménien. Héritage traumatique et travail analytique. 


\section{Notes}

1. Pour la question de la temporalité voir le chapitre «L'élaboration d'un héritage traumatique par déplacement dans le temps » dans J. Altounian, 2012.

2. Cette traduction avait été, en 1982, remise par le traducteur aux Temps modernes sous forme dactylographiée ; la version, établie par la revue, avait été plus tard reproduite par les Belles Lettres dans J. Altounian, 1990.

3. Il faut rappeler ici que, sur son versant oriental, la Grande Guerre a servi de paravent à la perpétration du génocide arménien de 1915 par les Jeunes Turcs, alors alliés des puissances centrales, l'Autriche et notamment l'Allemagne qui leur apporta un important soutien.

4. Goethe, Faust I, vers 682/4 :

Was du ererbt von deinen Vätern hast,

Erwirb es, um es zu besitzen.

Was man nicht nützt ist eine schwere Last.

5. Sur les questions d'histoire, voir Leslie A. Davis, 1915, La province de la mort, Archives américaines concernant le génocide des Arméniens, Bruxelles, Complexe, 1994 ; Vahakn Dadrian, 1996, Histoire du génocide arménien, Paris, Stock; Yves Ternon, 1996, Les Arméniens, histoire d'un génocide, Points Histoire, Paris, Seuil; G. Bensoussan, C. Mouradian, Y. Ternon (dir.), 2003, Ailleurs, hier, autrement: connaissance et reconnaissance du génocide des Arméniens, Revue d'histoire de la Shoah, $\mathrm{n}^{\text {os }}$ 177-178; Raymond Kévorkian, 2006, Le génocide des Arméniens, Paris, Odile Jacob/Histoire; Claire Mouradian et Anouche Kunth, 2010, Les Arméniens en France, Du chaos à la reconnaissance, Paris, Collection Exils, Toulouse, Éditions de l'attribut; Yervant Odian, 2010, Journal de Déportation, traduit par Léon Ketcheyan, préface de Krikor Beledian, Marseille, Parenthèses; F. Cetin, 2004, Le livre de ma grand mère, traduit du turc par A. Krikorian et L. Djolokian, La Tour d'Aigues, Éditions de l'Aube, 2006; A. G. Altinay F. et Cetin, 2009, Les petits-enfants, traduit par Célin Vuraler, Paris, Actes Sud, 2011 ; Laurence Ritter et Max Sivaslian, 2012, Les restes de l'épée: les Arméniens cachés et islamisés de Turquie, Paris, Thaddée; Laure Marchand et Guillaume Perrier, 2013, La Turquie et le fantôme arménien. Sur les traces du génocide, Paris, Solin.

6. Devant la poursuite des massacres, en 1896, dans les régions de Van, Mouch, Killis et Egin, le parti révolutionnaire Dachnak cherchait un moyen d'obliger les puissances alliées à intervenir. Le mercredi 26 août 1896 à 13 heures, les Dashnaks prirent possession de la Banque ottomane, où prédominaient les investissements britanniques et français.

7. Au sens exact du «Einfall» freudien, traduit dans les OCF/P, PUF, par «idée incidente» ou «idée qui vient».

8. L'accueil de ce manuscrit par cette revue avait été évidemment favorisé par celui de trois articles précédents: "Comment peut-on être Arménien? », Les Temps Modernes, décembre 1975, n 353; «Une Arménienne à l'école», Les Temps Modernes, août/septembre $1977, n^{\text {os }} 373 / 374$; «À la recherche d'une relation au père, soixante ans après un génocide", Les Temps Modernes, décembre 1978, nº 389 (Ensemble repris également dans J. Altounian, 1990).

9. Les Euvres complètes de Freud/Psychanalyse (directeurs de la publication: André Bourguignon - Pierre Cotet, Directeur scientifique: Jean Laplanche) dont 18 volumes ont paru depuis le premier en 1988 aux Presses universitaires de France, les deux derniers étant à paraître fin 2013 et 2014 . 


\section{Références}

Altounian, J., 1990, «Ouvrez-moi seulement les chemins d'Arménie », Un génocide aux déserts de conscient (préface de René Kaës), Paris, Les Belles Lettres/Confluents psychanalytiques.

Altounian, J., 2000, La survivance/Traduire le trauma collectif (préface de Pierre Fédida, Postface de René Kaës), Paris, Dunod/Inconscient et Culture.

Altounian, J., 2001, L'école de la République, jadis «mère adoptive » pour les sinistrés, l'est-elle encore?, Les Temps Modernes, ${ }^{\text {os }}$ 615-616/septeptembre-octobre-novembre.

Altounian, J., 2003, L'écriture de Freud. Traversée traumatique et traduction, Paris, PUF, bibliothèque de psychanalyse.

Altounian, J., 2005, L'intraduisible. Deuil, mémoire, transmission, Paris, Dunod/Psychismes.

Altounian, J., 2012, De la cure à l'écriture/L'élaboration d'un héritage traumatique, Paris, PUF.

Altounian, J., et Altounian, V. (con un saggio di Manuela Fraire), 2007, Ricordare per Dimenticare. Il genocidio armeno nel diario di un padre e nella memoria di una figlia, Roma, Donzelli Editore, Saggine/107.

Altounian, V., et Altounian, J. (avec la contribution de Beledian, K., Chiantaretto, J.F., Fraire, M., Gampel, Y., Kaës, R., et Waintrater, R.), 2009, Mémoires du Génocide arménien. Héritage traumatique et travail analytique, Paris, PUF.

Altounian, V., 1982, Journal «Tout ce que j’ai enduré des années 1915 à 1919», (traduction, notes et postface de Krikor Beledian), intitulé par J. Altounian «Terrorisme d'un génocide», Les Temps Modernes, $n^{\circ}$ 38/427, repris in Altounian, J., 1990, «Ouvrez-moi seulement les chemins d'Arménie», Un génocide aux déserts de conscient, Paris, Les Belles Lettres, 96-100; et dans une version révisée dans Altounian, V., et Altounian, J., Mémoires du Génocide arménien. Héritage traumatique et travail analytique, Paris, PUF, 13-41.

Beledian, K., 1982, postface du Journal «Tout ce que j'ai enduré des années 1915 à 1919 », intitulé par J. Altounian «Terrorisme d'un génocide», Les Temps Modernes, n 38/427,

Beledian, K., 2009, Traduire un témoignage écrit dans la langue des autres, in Altounian, V., et Altounian, J., Mémoires du Génocide arménien. Héritage traumatique et travail analytique, Paris, PUF.

Benjamin, W., 1933, Erfahrung und Armut, Expérience et pauvreté, Euvres II, traduit par de Gandillac M., Rochlitz R., Rusch P., Paris, Gallimard, collection Folio Essais, 2000.

Benjamin, W., 1940, Über den Begriff der Geschichte, Sur le concept d'histoire, Euvres III, traduit par de Gandillac M., Rochlitz R., Rusch P., Paris, Gallimard, collection Folio Essais, 2000.

Freud, S., 1936, Lettre à Romain Rolland (Un trouble du souvenir sur l'Acropole), in OCF/P XIX, Paris, PUF, 1995, 331-338.

Freud, S., 1912, Totem et tabou, in OCF/P XI, Paris, PUF, 1998.

Jurgenson, L., 2003, L'expérience concentrationnaire, est-elle indicible?, Paris, Éditions du Rocher.

Jurgenson, L., L'expérience concentrationnaire, Entretien avec Luba Jurgenson par Alexandre Prstojevic, site de Vox Poetica, http://www.vox-poetica.org/entretiens/intJurgenson.html.

Kaës, R., 1993, Le sujet de l'héritage, in Transmission de la vie psychique entre générations, Paris, Dunod/Inconscient et culture.

Kaës, R., 2009, Le travail de l'intersubjectivité et la polyphonie du récit dans l'élaboration de l'expérience traumatique, in Altounian, V., et Altounian, J., Mémoires du Génocide arménien. Héritage traumatique et travail analytique, Paris, PUF.

Trévisan, C., 2009, Questions de communication, Nancy, Presses universitaires de Nancy. Waintrater, R., 2003, Sortir du génocide. Témoigner pour réapprendre à vivre, Paris, Payot. 